\title{
Serum thymidine kinase 1 is a reliable maker for the assessment of the risk of developing malignancy: A case report
}

\author{
ZHIHENG CHEN ${ }^{1}$, HONG GUAN $^{2}$, HONG YUAN $^{3}$, XIA CAO $^{1}$, \\ YINGXIN LIU ${ }^{1}$, JI ZHOU ${ }^{4}$, ELLEN HE ${ }^{4}$ and SVEN SKOG ${ }^{4}$ \\ ${ }^{1}$ Health Management Centre, Third Xiangya Hospital, Central South University, Changsha, Hunan 410013; ${ }^{2}$ Department of \\ Pathology, Shenzhen Second Hospital, Shenzhen, Guangdong 322000; ${ }^{3}$ Research Center of Sub-Health's Diagnose and \\ Intervention Technology and Department of Cardiology, The Third Xiangya Hospital, Central South University, Changsha, \\ Hunan 410013; ${ }^{4}$ Sino-Swed Molecular Bio-Medicine Research Institute, Shenzhen, Guangdong 518057, P.R. China
}

Received September 10, 2014; Accepted June 2, 2015

DOI: $10.3892 / \mathrm{ol} .2015 .3440$

\begin{abstract}
With regard to different types of malignancies, thymidine kinase 1 (TK1) is a useful prognostic marker in clinical oncology, both as a serum proliferation marker and in immunohistochemistry. The present study investigated the use of serum TK1 protein (STK1p) for the identification of multiple proliferating diseases linked to the risk of developing cancer, by following one patient during the period of 2003-2014. The patient presented with adenomatous polyps in the stomach in 2003, follicular cervicitis in 2007 and hyperplasia of the breast/fibrocystic breasts in 2010 . The breast cysts increased from 4x5 mm in size in 2010 to $8 \times 7 \mathrm{~mm}$ in size in 2013, and were assessed as a suspicious malignancy at the end of this period. In parallel, the STK1p values increased from 2.0 to $7.6 \mathrm{pM}$. Based on this information, a minimally invasive surgery using the Mammotome ${ }^{\circledR}$ Biopsy System was performed. Immunohistochemistry on the cyst tissue showed strong staining of TK1 in the ductal epithelial cells and thus confirmed the abnormal proliferation in the lesion. One week after the surgery, the STK1p value had decreased to almost normal values (1.6 pM), but then fluctuated above $2.0 \mathrm{pM}$ for the next 7 months. After the surgery, the patient was re-examined and small foci with squamous cell hyperplasia and a suspected ulcerated cervix, as well as flat gastric erosive, were identified, but not treated; this may explain why the STK1 P-values did not return to within normal values. The patient is currently being followed
\end{abstract}

Correspondence to: Professor Sven Skog, Sino-Swed Molecular Bio-Medicine Research Institute, 19F, A2 Building, Gaoxin C, 1st Avenue, Shenzhen, Guangdong 518057, P.R. China

E-mail: svenisak@icloud.com

Professor Zhiheng Chen, Health Management Centre, Third Xiangya Hospital, Central South University, 138 Tong Zi Po Road, Changsha, Hunan 410013, P.R. China

E-mail: 873127193@qq.com

Key words: thymidine kinase 1 , serum thymidine kinase 1 protein, proliferation, precancerous, health screening up using STK1p analysis combined with imaging/pathology in order to begin therapeutic intervention as early as possible to avoid the risk of developing cancer. Overall, STK1p is useful in health screening to identify individuals at risk of developing premalignancy/malignancy.

\section{Introduction}

Cancer is a disease that is characterized by the proliferation of abnormal cells. Mutations in specific proteins and enzymes that are associated with the regulation of cell growth results in uncontrolled proliferation and thus malignancy. In recent decades, the variety of cancer treatment methods (surgery, radiotherapy, chemotherapy and endocrine therapy) have been markedly improved, however, cancer remains the leading cause of mortality in China. Approximately $98 \%$ of individuals who undergo health screening exhibit diseases or illness of different types, $50 \%$ of which are linked to the development of premalignancies or malignancies $(1,2)$, indicating that health screening is an important element for improving the quality of life.

Thymidine kinase 1 (TK1) is an enzyme that is important for the regulation of the intracellular thymidine pool throughout the cell cycle. The TK1 enzyme is worthy of note as its level is highly dependent on the growth stage of the cell. In proliferating normal and tumor cells, the level of TK1 starts to increases at late $\mathrm{G}_{1}$ phase, and reaches a maximum in late $S$-phase/early $G_{2}$ phase of the cell cycle, but in quiescent cells, TK1 is almost completely absent. This singles out TK1 as a useful indicator of cellular proliferation, and hence for malignancy (3-6). Serum TK1 protein (STK1p) level, determined by non-invasive serological methods, was found to be an emerging potential cell proliferation biomarker for the prognosis of cancer patients (7-9), for monitoring tumor treatments, relapse, follow-up and survival, and particularly for the early detection of cancer development risk $(10,11)$. A sensitive chemiluminescence dot blot assay of STK1p was developed by the Swedish TK1 Research Team in 2000 (7,9), which has become a commercial kit (Sino-Swed Biotech Ltd., Shenzhen, China) for the early detection of pre/early risk of developing cancer in health screening $(1,2,12)$. Using this kit, a 
health screening study on 35,365 individuals showed a receiver operating characteristic value of 0.96 for the STK1p assay. At a cut-off STK1p value of $2.0 \mathrm{pM}$, the likelihood (+) value was 236.5 , and the sensitivity and the specificity were 0.78 and 0.99 ( 1 out of 300 false-positives), respectively, indicating that the STK1p assay is a reliable test for the risk assessment of the pre/early cancerous progression of individuals in health screening (13).

The current study presents the case of a patient that exhibited a higher risk of developing malignancies for three types of diseases. This patient was followed up for 139 months using the STK1p assay (last 83 months) in combination with imaging, histological and immunological techniques, and routine clinical laboratory tests.

\section{Case report}

Patient. The patient was a 52-year-old premenopausal female with regular menstruation. The patient works at a medical hospital as a nurse in a health-screening center, is married with one daughter, and has undergone an abortion twice. No family history of cancer and no history of exposure to any environmental risk factors were recorded. The patient provided written informed consent for participation in the present study.

Three types of proliferating diseases. The case study was performed between January 2003 and July 2014 (139 months). At least three types of proliferating diseases linked to cancer development risk were found: i) Gastric diseases detected by gastroscopy and biopsy; ii) cervical disease detected by cervical human papilloma virus (HPV)-DNA detection, thinprep cytological test, colposcopy and biopsy; and iii) breast proliferative tissue detected by automated breast volume scan (ABVS) and color Doppler ultrasound scan.

Health screening tests. The health-screening included an ear, nose and throat physical examination, blood pressure tests, liver, gallbladder, spleen, pancreas, double kidney and thyroid analysis by ultrasound, blood and biochemical tests, analysis of urine routine, thyroid function and sex hormone levels, a oral glucose tolerance test, and analysis of rheumatoid and tumor markers (cancer antigen (CA)125, CA153, $\alpha$-fetoprotein and carcinoembryonic antigen). These types of tests were performed annually between 2007 and 2014 (83 months).

STK1 assay. The STK1p test was included in the annual health screening of the patient in 2008 and used until 2014 (83 months).

The STK1p assay was performed using a commercial kit, based on an enhanced chemiluminescence dot blot assay (SSTK Biotech Ltd., Shenzhen, China) (13). Briefly, $3 \mu$ l serum were directly applied onto a nitrocellulose membrane. The serum samples were probed with human anti-TK1 chicken immunoglobulin Y antibody. Varying concentrations of TK1-pepetide (20, 6.6 and 2.2 pM) were used as an extrapolation standard. The spot intensities on the membrane were determined by a CIS-1 Imaging System (SSTK Biotech Ltd.). From the intensities of the TK1 standard of known concentrations, the STK1 concentration was calculated and expressed in $\mathrm{pM}$. The coefficient of variation was $<10 \%$. The threshold value of STK1 was set to $2.0 \mathrm{pM}$. STK1p values of $<2.0 \mathrm{pM}$ were denoted as normal, considered as a lower risk for developing malignancy. STK1p values $>2.0 \mathrm{pM}$ were denoted as elevated, likely representing individuals with an increased risk of pre-malignancy/malignancy progression.

TK1 immunohistochemical staining. TK1 immunohistochemical staining was performed using the EnVision System according to the manufacturer's instructions (Maxin Biotech, Fuzhou, China), as previously described $(10,14)$. In brief, two serial sections were used for the staining of the breast tissue with human TK1 monoclonal antibody (dilution, 1:800 in PBS; stock concentration, $1 \mathrm{mg} / \mathrm{ml}$; SSTK Biotech. Ltd., Shenzhen, China). The quality of the TK1 monoclonal antibody used in this study was also confirmed by independent research groups. At least 100 cells were counted in $~ 10$ light microscopic fields at $\mathrm{x} 400$ magnification.

The present study was approved by the Committee of Research Ethics at the Third Xiangya Hospital (Central South University, Changsha, Hunan, China). The patient provided written informed consent to participate in this study, which was conducted in accordance with the Helsinki Declaration of 1983.

\section{Test results}

Health screening tests. The physical examination and the biochemical routine tests, excluding the STK1p assay, showed normal values.

Gastric diseases. The patient had experienced a gastrointestinal problem with stool shapeless for $>20$ years. In January 2003, using gastroscopy and a biopsy of the gastric tissue, an adenomatous polyp was detected, but no gastric ulcer. In June 2014, the gastric adenomatous polyp developed into a small foci with flat gastric erosive and inflammation of the cardia (Fig. 1). No treatment for these changes was performed.

Cervix diseases. The patient had been affected by chronic follicular cervicitis since August 2007. The pathological changes were found based on several tests with cervical HPV-DNA detection and TCT. However, in July 2014, aside from the chronic follicular cervicitis previously found in 2007 , small foci with squamous cell hyperplasia and suspected ulcerated cervix were found using colposcopy and biopsy of the cervix (Fig. 1). No treatment was performed for this type of changes.

Breast diseases. In March 2010, using the ABVS and color Doppler ultrasound scan, a breast hypoechoic nodule was found in the right breast $(4 \times 5 \mathrm{~mm})$ (Fig. 1). The patient was then followed up with ultrasound several times. The size of the breast hypoechoic nodule increased to $8 \times 7 \mathrm{~mm}$ after 45 months (December 2013). The ABVS and color Doppler ultrasound scan showed inhomogeneous breast proliferative tissues (Fig. 2). A pixel pitch of 8x7 mm showed a hypoechoic focus with a circumscribed and irregular shape, $7 \mathrm{~mm}$ under the skin surface, as evaluated as Breast Imaging Reporting and Data System category 3 (BI-RADS 3, American College of Radiology, 1998; Fig. 2). The color Doppler ultrasound scan of the right breast revealed that the mammary glands were coarsely heterogeneous (Fig. 2), indicating a suspicious malignancy. 


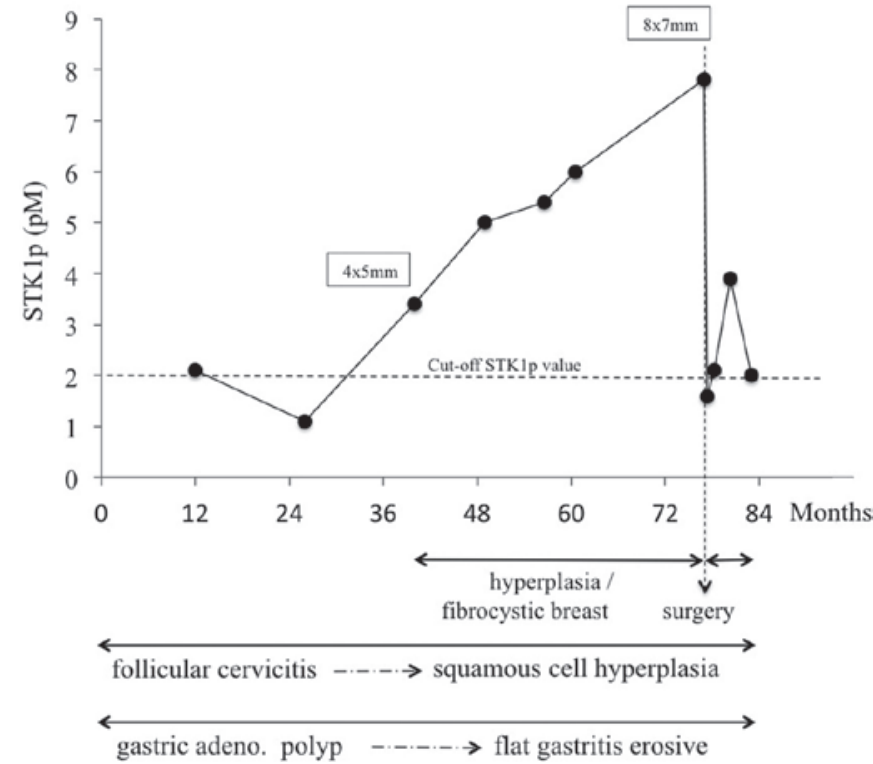

Figure 1. Summary of the study, including histopathology and STK1p values during the follow-up period of 84 months. The health screening started in 2007 (month 0) The values in the boxes are the size of the fibrocysts in the breast. STK1p, serum thymidine kinase 1 protein.
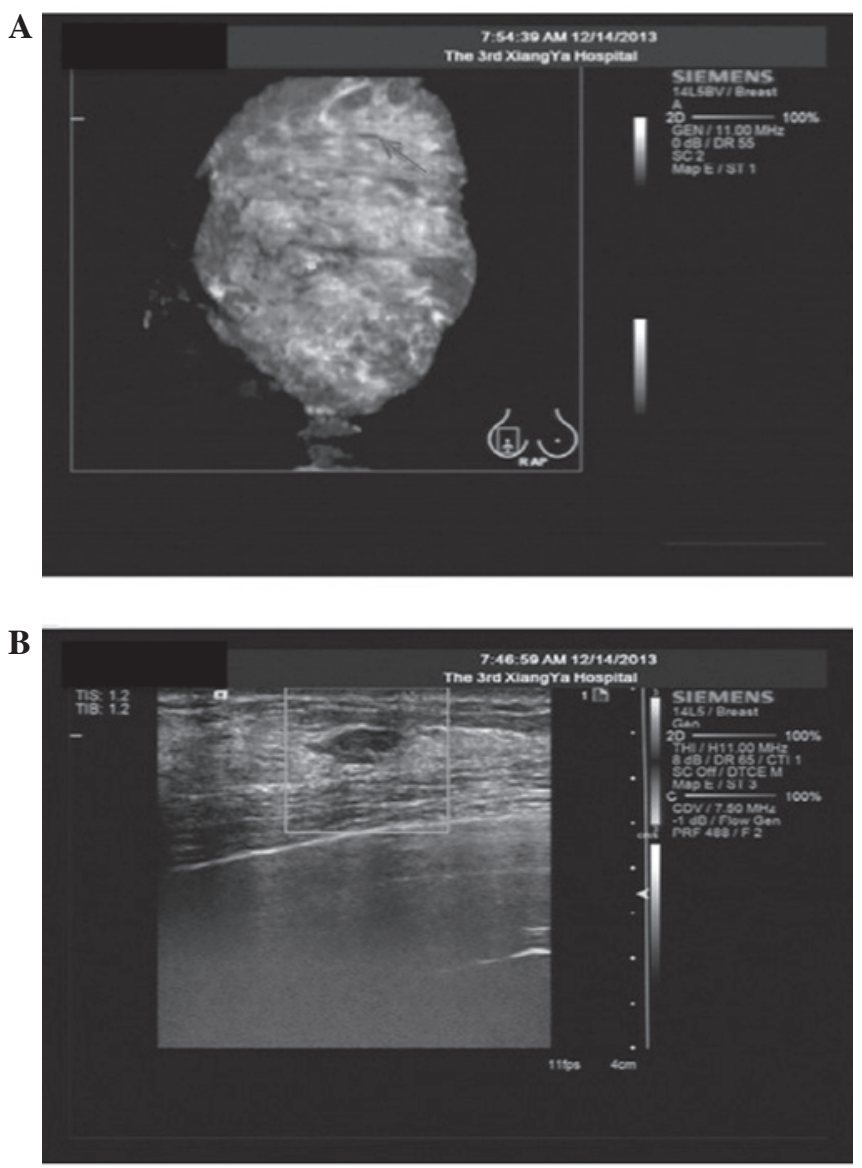

Figure 2. Automated breast bolume scan (ABVS) and color Doppler ultrasound scan. (A) Screening ABVS of the right breast in three orthogonal planes showing coronal reconstruction, axial plane and sagittal reconstruction, revealing inhomogeneous breast proliferative tissues. A pixel pitch of $0.8 \times 0.7 \mathrm{~mm}$ showing a hypoechoic focus with a circumscribed and irregular shape was found $7 \mathrm{~mm}$ under the skin surface (Breast Imaging Reporting and Data System category 3). (B) Color Doppler ultrasound scan of the right breast revealing coarsely heterogeneous mammary glands.
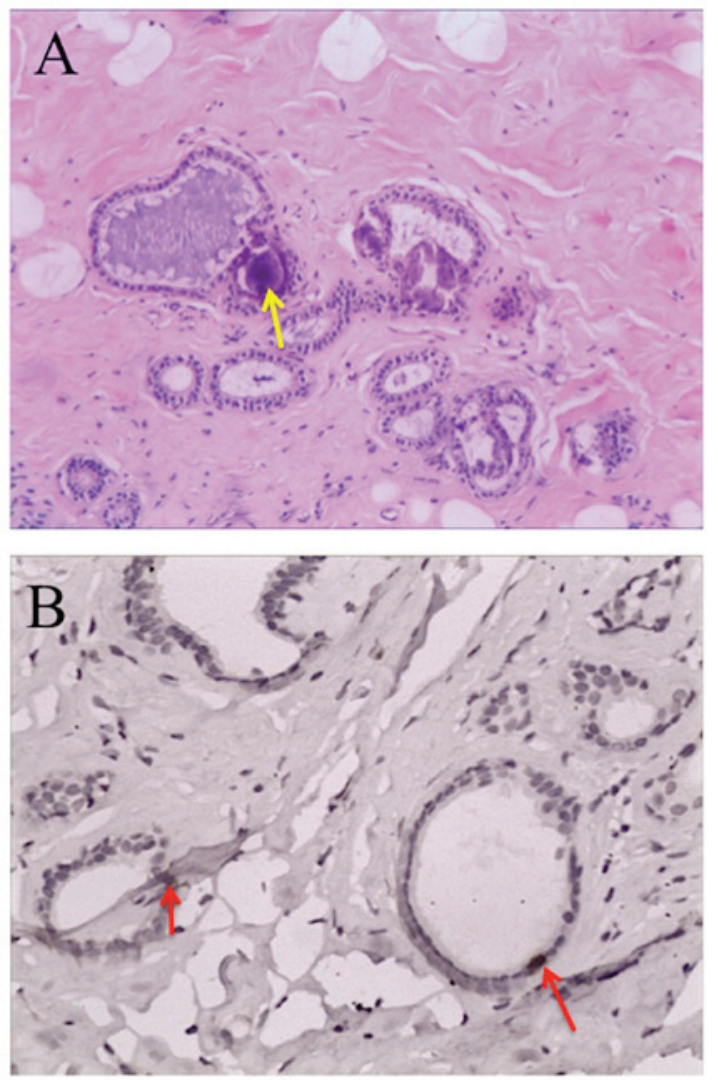

Figure 3. (A) Fibrocystic breast disease. Hematoxylin staining showing cystic expansion of proliferating cells (yellow arrow). (B) Example of TK1-positive staining in ductal epithelial cells (red arrow).

Pre-operative STK1p. The changes in the levels of STK1p are summarized in Fig. 1. In January 2008, STK1p analysis was included in the annual health screening, revealing a fluctuating STK1p value of between 1.2 and $2.1 \mathrm{pM}$. The STK1p value of healthy individuals without any known diseases or illness is $<1.0 \mathrm{pM}(1,2,11,12)$.

In March 2010, the STK1p value was elevated (3.4 pM) and continued to increase up to $7.8 \mathrm{pM}$ in December 2013 (Fig. 1). The increase in the STK1p value was parallel with the discovery of hypoechoic nodules in the right breast, as determined by ultrasound examination (Fig. 2). Based on the hypoechoic nodules and the high STK1p value (7.8 pM), surgery to remove the nodules was performed on December 20, 2013, using minimally invasive surgery with the Mammotome ${ }^{\circledR}$ Biopsy System (Mammotome, Cincinnati, OH, USA). Histopathological investigations on the hypoechoic nodules showed an expanding fibrocyst type, but no evidence of cancer (Fig. 3A). In addition, TK1 immunohistochemistry showed strong positive staining in the ductal epithelial cells (Fig. 3B).

Post-operative STK1p. Following surgery, the BI-RADS scan did not show any significant mass in the breast. The patient did not undergo any type of additional treatment. The STK1p values declined to nearly normal values $(1.6 \mathrm{pM})$ one week after surgery. The STK1p levels, however, followed a somewhat increasing during the following 7 months, fluctuating around $2.0 \mathrm{pM}$ (Fig. 1). 


\section{Discussion}

Premalignancies and long-term chronic diseases are linked to the risk of the development of malignant diseases. Cancer-related lifestyle risk factors, such as physical activity, smoking and eating habits, play a primary role in the etiology of cancer $(15,16)$. Premalignancies are easier to treat than malignancies and therefore, early screening is a cost-effective cancer prevention strategy. Premalignancy treatment is also responsible for the most marked reduction in cancer-related mortality attributable to any medical intervention $(17,18)$. The detection of premalignancies with routine screening could prevent the occurrence of the majority of human cancers (11). However, the majority of tumor biomarkers are often insufficient to diagnose specific types of malignancies and as a result, are not recommended for the screening of early cancer diseases (19). Tumors of a small size are now identified by in vivo imaging techniques, but these techniques are expensive. Furthermore, such imaging techniques cannot differentiate premalignant lesions from malignant lesions that well. The imaging technique is also limited regarding a premalignancy or extremely small malignant tumors. Premalignancies are the earliest morphological discernible lesions that precede the development of carcinoma in situ. Numerous premalignancies can be identified by pathology $(17,20)$. However, it is not easy to assess the proliferation rate. In the present study, STK1p combined with TK1 immunohistochemical staining was shown to be a reliable biomarker for the assessment of abnormal proliferation in the lesion, indicating the presence of physiological changes associated with the development of premalignancies.

A previous study on healthy individuals without any tumor diseases showed that 99\% exhibited a STK1p level of $<1.0 \mathrm{pM}$ (1). In cancer patients, the STK1p values correlate to tumor cell proliferating rates of different levels $(5,9,11)$. Patients are considered to be tumor-free when the STK1p values were normalized $(<1.0 \mathrm{pM})$ within 1-3 months after the end of the treatment. Thus, a close correlation has been found between the STK1p values and clinical parameters, including the outcome of tumor treatments $(10,11)$.

In the present study, a close correlation was found between the development of breast nodes and the STK1p value, and a decline was observed in the STK1p values from high to almost normal values following minimally invasive surgery using the Mammotome Biopsy System (Fig. 1). These results confirmed that STK1p is a useful serum proliferation biomarker in health screening and in monitoring the treatment.

Beside the usefulness of STK1p, TK1 immunohistochemical staining in combination with histology is also a useful tool for the assessment of the risk of developing cancer. As shown in Fig. 3, the fibrocystic breast disease developed into cystic expansion of proliferating cells in parallel with strong TK1 staining of ductal epithelial cells, indicating a risk of developing malignancy (20).

Subsequent to minimally invasive surgery, the STK1 values tended to increase, fluctuating around $2.0 \mathrm{pM}$ (Fig. 1). The reason for this could be that the changes in the adenomatous gastric polyps and the chronic follicular cervicitis over time into more advanced types were not treated. These changes also indicate that premalignancies were developing (20). The patient is currently being followed up by STK1p combined with imaging and pathology in order to begin therapeutic intervention as early as possible to avoid the risk of developing cancer.

In conclusion, STK1p and TK1 immunohistochemistry in combination with imaging and pathology are useful for the early detection of premalignancy processes and for the early risk warning of invisible tumors.

\section{Acknowledgements}

This study was made possible by grants from the Health Management Centre of the Third Xiangya Hospital and the Department of Pathology (Shenzhen Second Hospital, Shenzhen, Guangdong, China). The authors would also like to thank Sino-Swed Tongkang Biotech. Ltd., for providing technical support.

\section{References}

1. Chen Z, Zhou H, Li S, He E, Hu J,Zhou J and Skog S: Serological thymidine kinase 1 (STK1) indicates an elevated risk for development of malignant tumours. Anticancer Res 28: 3897-3907, 2008.

2. Huang S, Lin J, Guo N, Zhang M, Yun X, Liu S, Zhou J, He E and Skog S: Elevated serum thymidine kinase 1 predicts risk of pre/early cancerous progression. Asian Pac J Cancer Prev 12: 497-505, 2011.

3. Sherley JL and Kelly TJ: Regulation of human thymidine kinase during the cell cycle. J Biol Chem 263: 8350-8358, 1988.

4. Kauffman MG and Kelly TJ: Cell cycle regulation of thymidine kinase: Residues near the carboxyl terminus are essential for the specific degradation of the enzyme at mitosis. Mol Cell Biol 11: 2538-2546, 1991

5. He Q, Zhang P, Li Z, Li H, Wang X, Zou S, Fornander T and Skog S: Concentration of thymidine kinase 1 in serum (S-TK1) is a more sensitive proliferation marker in human solid tumors than its activity. Oncol Rep 14: 1013-1019, 2005.

6. Welin M, Kosinska U, Mikkelsen NE, Carnrot C, Zhu C, Wang L, Eriksson S, Munch-Petersen B and Eklund H: Structures of thymidine kinase 1 of human and mycoplasmic origin. Proc Natl Acad Sci USA 101: 17970-17975, 2004.

7. He Q, Zou L, Zhang PA, Liu JX, Skog S and Fornander T: The clinical significance of thymidine kinase 1 measurement in serum of breast cancer patients using anti-TK1 antibody. J Biol Marker 15: 139-146, 2000.

8. Wu CJ, Yang R, Zhou J, Bao S, Zou L, Zhang P, Mao Y, Wu J and He Q: Production and characterisation of a novel chicken IgY antibody raised against C-terminal peptide from human thymidine kinase 1. J Immuno Methods 277: 157-169, 2003.

9. He Q, Fornander T, Johansson H, Johansson U, Hu GZ, Rutqvist LE and Skog S: Thymidine kinase 1 in serum predicts increased risk of distant or loco-regional recurrence following surgery in patients with early breast cancer. Anticancer Res 26: 4753-4759, 2006.

10. Aufderklamm S, Todenhöfer T, Gakis G, Kruck S, Hennenlotter J, Stenzl A and Schwentner C: Thymidine kinase and cancer monitoring. Cancer Lett 316: 6-10, 2012.

11. Zhou J, He E and Skog S: The proliferation marker thymidine kinase 1 in clinical use. Mol Clin Oncol 1: 18-28, 2013.

12. Xu XH, Zhang YM, Shu XH, Shan LH, Wang ZW, Zhou YL, Wen HK, He F, He E and Skog S: Serum thymidine kinase 1 reflects the progression of pre-malignant and malignant tumors during therapy. Mol Med Rep 1: 705-712, 2008.

13. Chen ZH, Huang SQ, Wang Y, Yang AZ, Wen J, Xu XH, Chen Y, Chen QB, Wang YH, He E, et al: Serological thymidine kinase 1 is a biomarker for early detection of tumours-a health screening study on 35,365 people, using a sensitive chemiluminescent dot blot assay. Sensors(Basel) 11: 11064-11680, 2011.

14. Guan H, Sun Y, Zan Q, Xu M, Li Y, Zhou J, He E, Eriksson S, Wen W and Skog S: Thymidine kinase 1 expression in atypical ductal hyperplasia significantly differs from usual ductal hyperplasia and ductal carcinoma in situ: A useful tool in tumour therapy management. Mol Med Reports 2: 923-929, 2009. 
15. Ang YK, Mirnalini K and Zalilah MS: A workplace email-linked website intervention for modifying cancer-related dietary and lifestyle risk factors: Rationale, design and baseline findings. Malays J Nutr 19: 37-51, 2013.

16. Sulaiman S, Shahril MR, Wafa SW, Shaharudin SH and Hussin SN: Dietary carbohydrate, fiber and sugar and risk of breast cancer according to menopausal status in malaysia. Asian Pac J Cancer Prev 15: 5959-5964, 2014.

17. Berman JJ and Moore GW: Part I, Limitation of Current Approaches to Cancer Treatment; Part II, Precancer Pathology and Biology; Part III, Eradication of Cancer By Treatment of Precancer. In: Precancer: The Beginning and the End of Cancer Jones and Bartlett Publishers, Sudbury, MA, USA, pp1-113, 2010
18. Pandey S and Chandravati: Breast screening in north India: A cost-effective cancer prevention strategy. Asian Pac J Cancer Prev 14: 853-857, 2013.

19. Cigna: Tumor markers for cancer. http://cignaforhcp.cigna.com/ public/content/pdf/coveragePolicies/medical $/ \mathrm{mm}$ _0172_coverage positioncriteria_tumor_markers_for_diagnosis_mgmt_cancer.pdf. Accessed on January $1,2015$.

20. Underwood JCE (ed): Classification of tumours. In: General and Systematic Pathology. Elsevier Ltd., pp227-229, 240, 235, 374, 473 and 497, 2004. 